\title{
Dose-dependent effects of intravenous methoxamine infusion during hip-joint replacement surgery on postoperative cognitive dysfunction and blood TNF-a level in elderly patients: a randomized controlled trial
}

Shenghui Sun ${ }^{1}$, Defeng Sun ${ }^{2 *}$, Lin Yang ${ }^{3}$, Jun Han ${ }^{2}$, Ruochuan Liư ${ }^{2}$ and Lijie Wang ${ }^{2}$

\begin{abstract}
Background: Postoperative cognitive dysfunction (POCD), common in elderly patients, is thought to be closely associated with intraoperative instability of hemodynamics and excessive excretion of tumor necrosis factor-a (TNF-a). Methoxamine is a blood-pressure increasing drug commonly used for maintaining intraoperative hemodynamics. Methoxamine potentially promotes TNF-a expression, leading to an increased risk of POCD. This study aimed to investigate the dose-dependent effect of methoxamine on the incidence of early POCD and blood TNF-a level.

Methods: This single-center prospective double-blind controlled clinical trial included a total of 300 adult patients (75-90 years old, American Society of Anesthesiologists class II-III) who underwent unilateral hip-joint replacement surgery under epidural anesthesia. Patients were randomly divided into three methoxamine groups (M1, M2, and M3), and one control group ( $n=75$ per group). During surgery, M1, M2, and M3 patients received intravenous infusion of methoxamine at 2, 3, or $4 \mu \mathrm{g} \cdot \mathrm{kg}^{-1} \cdot \mathrm{min}^{-1}$, respectively; the control group received saline of same volume at the same infusion rate. All patients received standard transfusion to maintain stable circulation. Hemodynamics, cardiovascular events, and serum TNF-a levels were monitored. Mini Mental State Examination was performed both before and after surgery to diagnose POCD.

Results: The primary outcome of this study was the incidence of POCD, which was higher in the M3 group (18.7\%) than in the control group (5.3\%), the M1 group (6.7\%), or the M2 group (6.7\%) (all $P<0.05$ ). The secondary outcomes were the postoperative blood TNF-a level and intraoperative hemodynamic parameters. The postoperative TNF-a level was found to be higher than baseline in all groups and was highest in M3 patients $(P<0.05)$. The intraoperative hemodynamic parameters showed improved stability in the M1 and M2 groups compared with the control group. However, in the M3 group, abnormally increased intraoperative blood pressure, cardiac output, and systolic stroke volume were observed.

(Continued on next page)
\end{abstract}

\footnotetext{
* Correspondence: sundefengyl@163.com

${ }^{2}$ Department of Anesthesiology, The First Affiliated Hospital of Dalian Medical University, No.222 ZhongshanRoad, Xigang District, Dalian 116011, Liaoning, People's Republic of China

Full list of author information is available at the end of the article
} 
(Continued from previous page)

Conclusions: Intravenous infusion of methoxamine at $2-3 \mu \mathrm{g} \cdot \mathrm{kg}^{-1} \cdot \mathrm{min}^{-1}$ can maintain stable hemodynamics in elderly patients during epidural anesthesia for hip-joint replacement surgery, without increasing the incidence of POCD. Increasing the dose to $4 \mu \mathrm{g} \cdot \mathrm{kg}^{-1} \cdot \mathrm{min}^{-1}$ provided no further advantages but induced adverse effects on the intraoperative hemodynamics.

Trial registration: Chinese Clinical Trial Register (Unique identifier: ChiCTR-INR-15007607, retrospectively registered 18 Dec 2015).

Keywords: Methoxamine, Elderly patients, Hip-joint replacement surgery, Postoperative cognitive dysfunction, Tumor necrosis factor-a

\section{Background}

Postoperative cognitive dysfunction (POCD), common in the elderly, refers to a new cognitive impairment after surgery, especially decline in memory and executive functions [1]. POCD can lead to personality changes, social skill deficits, and emotional problems including anxiety [2-4]. POCD can significantly interfere with postoperative recovery, increasing patients' morbidity and mortality rates as well as hospital expenses [5, 6]. It often lasts from a few days to a few weeks after surgery, but has the potential to persist for or occur after several weeks to 7.5 years [7].

Although it is still unclear at present, the mechanism of POCD is considered to involve dysfunction of the central nervous, endocrine, and immune systems, caused by multiple factors associated with surgery and anesthesia [8]. The peripheral immune system can be activated by surgical injury, leading to the release of various inflammatory cytokines including tumor necrosis factor-alpha (TNF- $\alpha$ ), which can diffuse through the blood-brain barrier and cause cognitive deficits $[9,10]$. Furthermore, it has been demonstrated that beta-adrenoceptor activation suppresses TNF- $\alpha$ release, whereas alpha-adrenoceptor activation stimulates TNF- $\alpha$ release $[11,12]$.

Because epidural anesthesia is thought to be associated with a relatively low incidence of POCD, it is commonly used for hip-joint replacement surgery [13]. Elderly patients are the major recipients of hip-joint replacement. In patients older than 70 years, who often have compromised critical function and are highly sensitive to drugs, epidural anesthesia and the surgical procedure can induce significant physiological changes including disturbance of hemodynamics, hypotension, and bradycardia, especially in patients with high blood pressure and coronary artery disease $[14,15]$. Therefore, vasoactive drugs, including ephedrine and dopamine, are often used clinically to maintain stable hemodynamics during hip-joint replacement surgeries, which is important for the balance between oxygen supply and consumption in the myocardium and the prevention of pulmonary edema caused by fast or excessive fluid transfusion. However, ephedrine and dopamine can also increase the heart rate and myocardium oxygen consumption while increasing the blood pressure and therefore are commonly substituted with methoxamine in current practice $[16,17]$.

Methoxamine is a sympathomimetic drug that can directly activate peripheral vascular $\alpha_{1}$-adrenergic receptors, leading to vessel constriction and increases in both systolic and diastolic blood pressure. These increased blood pressures can further activate the carotid sinus baroreflex, decreasing the heart rate and myocardium oxygen consumption. Furthermore, methoxamine itself does not alter myocardium excitability or increase myocardium oxygen consumption even as it increases the blood flow in the coronary artery and endocardium $[17,18]$. Muscular or intravascular bolus injection of methoxamine often fails to maintain stable hemodynamics because of a delayed onset and short duration of action. In a previous study, we demonstrated that continuous intravenous infusion of methoxamine $\left(2 \mu \mathrm{g} \cdot \mathrm{kg}^{-1} \cdot \mathrm{min}^{-1}\right)$ can provide safe and effective maintenance of hemodynamics under epidural anesthesia during hip-joint replacement surgery in elderly patients [19].

In summary, POCD is thought to be closely associated with intraoperative instability of hemodynamics and excessive excretion of TNF- $\alpha$ [20]. Methoxamine is a blood-pressure increasing drug commonly used for maintaining intraoperative hemodynamics. Methoxamine potentially promotes TNF- $\alpha$ expression, leading to an increased risk of POCD. The primary aim of his study was to investigate the effect of intraoperative infusion of methoxamine at three different dosages of methoxamine on the incidence of early POCD and blood TNF- $\alpha$ level. The secondary aim of this study was to provide evidence for choosing the optimal methoxamine dosage that can maintain stable hemodynamics during surgery without increasing the risk of early POCD.

In the present study, we aimed to investigate whether improved management of hemodynamics through appropriate methoxamine administration could lead to a lower incidence of early POCD and observe how it affects blood TNF- $\alpha$ levels. Three different dosages of methoxamine were investigated. The results were expected to provide evidence for choosing the optimal methoxamine 
dosage that can maintain stable hemodynamics during surgery without increasing the risk of early POCD.

\section{Methods \\ Ethics and patients}

This study was preapproved by our institutional Research Ethics Committee in 2012 (Identification No.: KY2012-42) and then retrospectively registered with the internationally recognized Chinese Ethics Committee of Registering Clinical Trials in 2015 (Unique identifier: ChiCTR-INR-15007607). Written informed consent was obtained from each patient or his/her authorized representative(s) when he/she was in a critical physical condition or mentally challenged. Between January 1, 2013 and September 30, 2015, 141 male and 159 female patients $(n=300)$ underwent unilateral hip-joint replacement surgery under epidural anesthesia in our Hospital.

Inclusion criteria: (1) patient was aged between 75 and 90 years old; (2) patient had a physical status classification of American Society of Anesthesiologists (ASA) II or III (i.e., mild but definite systemic disease such as mild diabetes and chronic sinusitis, or severe systemic disease including severe diabetes and severe trauma); (3) patient was scheduled for unilateral hip-joint replacement surgery under epidural anesthesia.

Exclusion criteria: (1) Mini Mental State Examination (MMSE) score of <23 points; (2) obvious liver or kidney dysfunction; (4) history of mental illnesses or neurological diseases; (5) history of taking tranquilizers or antidepressants, or history of alcohol abuse; (6) serious audio-visual obstacles that affected communication; (7) an education level lower than junior high school; (8) blood electrolyte imbalance or hyperglycemia; (9) severe vision or hearing loss; and (10) recent treatments with sedatives, antidepressants, analgesics, or monoamine oxidase inhibitors.

\section{Interventions}

Patients were randomly assigned to four groups based on assignments found in the numbered envelopes, as follows: a control group receiving intravenous saline infusion (Group C, $n=75$ ) and three methoxamine groups receiving 2 , 3 , or $4 \mu \mathrm{g} \cdot \mathrm{kg}^{-1} \cdot \mathrm{min}^{-1}(10 \mathrm{~mL} / \mathrm{h})$ methoxamine (i.e., Groups M1, M2, and M3, $n=75$ in each group). Patients in the control group received an infusion of normal saline $(10 \mathrm{~mL} / \mathrm{h})$.

\section{Epidural anesthesia procedures and hemodynamic maintenance}

Patients were subjected to $8 \mathrm{~h}$ food deprivation and $2 \mathrm{~h}$ water deprivation before surgery, without preoperative treatment. Epidural anesthesia was performed by injecting $2 \%$ lidocaine ( $3 \mathrm{~mL}$ per patient) into the epidural space via a catheter secured at the L2-L3 vertebral interval while the patient was in a side-lying position. The radial artery was catheterized for real-time blood pressure monitoring. After the level of anesthesia reached the T6-T8 vertebral level, $1 \%$ ropivacaine $(5-10 \mathrm{~mL}$ per patient) was injected into the same catheter at the L2-L3 vertebral interval. The M1, M2, and M3 groups received an intravenous infusion of methoxamine (catalogue number: 120,901, Grand Pharma (China) Co. Ltd., Wuhan) at dosages of 2, 3 , and $4 \mu \mathrm{g} \cdot \mathrm{kg}^{-1} \cdot \mathrm{min}^{-1}(10 \mathrm{~mL} / \mathrm{h})$ respectively, whereas patients in the control group were infused with normal saline $(10 \mathrm{~mL} / \mathrm{h})$. To maintain stable circulation during surgery, sodium lactate Ringer's solution and 6\% hydroxyethyl starch $130 / 0.4$ in $0.9 \%$ sodium chloride (Voluven'; Fresenius KabiNorge A.S., Halden, Norway) were administered intravenously first at a rate of $0.5-1.0 \mathrm{~mL} \cdot \mathrm{kg}^{-1} \cdot \mathrm{min}^{-1}$ for $20-40 \mathrm{~min}$ and then maintained at $0.25 \mathrm{~mL} \cdot \mathrm{kg}^{-1} \cdot \mathrm{min}^{-1}$ with a crystal colloid ratio of 3:1. If the intraoperative blood pressure was lower than $70 \%$ of the baseline blood pressure (the reading at $10 \mathrm{~min}$ before anesthesia), the infusion rate was increased or ephedrine (5-6 mg) was administered intravenously. Depending on the surgical practice, $3-5 \mathrm{~mL}$ of $1 \%$ ropivacaine was added per patient as needed.

\section{Physiological monitoring during anesthesia}

During anesthesia preparation and surgery, patients were monitored by electrocardiogram and measurement of mean arterial blood pressure (MAP), central venous pressure $(\mathrm{CVP})$, heart rate $(\mathrm{HR})$, rate pressure product (RPP) (HR $\times$ systolic blood pressure), saturation of peripheral oxygen $\left(\mathrm{SpO}_{2}\right)$, cardiac output $(\mathrm{CO})$, and stroke volume (SV), using a Beneview T8 patient monitor (Shenzhen Mindray Bio-Medical Electronics Co., Ltd., Shenzhen, China) and the FloTrac Vigileo Sensor (Edwards Lifesciences LLC, USA). Values for these parameters were noted at $10 \mathrm{~min}$ before anesthesia (T1); $10 \mathrm{~min}$ (T2), $30 \mathrm{~min}$ (T3), and $1 \mathrm{~h}$ (T4) after anesthesia; and at the conclusion of surgery (T5). The radial artery and internal jugular vein were catheterized for intravascular monitoring of MAP and CVP. Low flow high-concentration oxygen (1-2 L/min) was delivered via face mask, resulting in an inspired oxygen concentration of $25 \%-29 \%$. Blood samples were collected from the nontransfused forearm ( $3 \mathrm{~mL}$ per patient) at $10 \mathrm{~min}$ before anesthesia, at the end of surgery, and at 1 and 2 days after surgery. The serum TNF- $\alpha$ level was measured using an enzyme-linked immunosorbent assay (ELISA). The total volume of liquid transfused, the dosage of ephedrine delivered during surgery, and the dosages of ropivacaine and sufentanil during and after surgery were noted. Urine volumes during surgery and $24 \mathrm{~h}$ after surgery were quantified. The incidences of pulmonary edema, high blood pressure $(30 \%$ higher than 


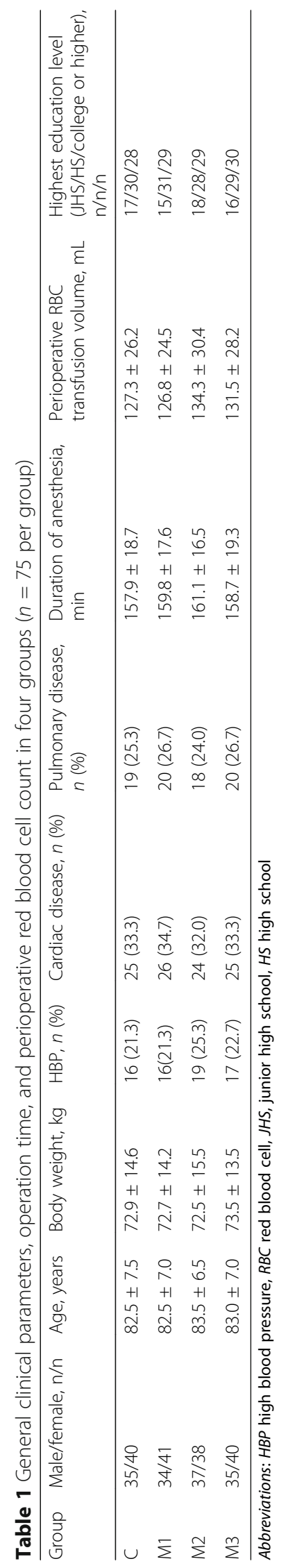


Table 2 Hemodynamic parameters at different time points in four groups ( $n=75$ per group)

\begin{tabular}{|c|c|c|c|c|}
\hline & C & M1 & M2 & M3 \\
\hline \multicolumn{5}{|c|}{ Mean arterial pressure (MAP) (mmHg) } \\
\hline Baseline & $89.7 \pm 9.2$ & $91.8 \pm 9.3$ & $91.5 \pm 9.1$ & $90.7 \pm 8.9$ \\
\hline 10 min_Anesthesia & $69.7 \pm 6.1^{a}$ & $87.5 \pm 8.5^{c}$ & $90.5 \pm 9.0^{c}$ & $92.8 \pm 9.1^{c}$ \\
\hline 30 min_Anesthesia & $71.5 \pm 6.7^{\mathrm{a}}$ & $88.1 \pm 9.3^{c}$ & $92.8 \pm 9.3^{c}$ & $94.4 \pm 9.2^{c}$ \\
\hline 60 min_Anesthesia & $72.8 \pm 7.4^{\mathrm{a}}$ & $90.2 \pm 9.5^{c}$ & $97.7 \pm 9.6^{c}$ & $111.9 \pm 13.4^{\text {acef }}$ \\
\hline End of operation & $75.9 \pm 8.1^{a}$ & $93.3 \pm 10.3^{c}$ & $98.3 \pm 10.5^{c}$ & $115.7 \pm 13.6^{\text {acef }}$ \\
\hline \multicolumn{5}{|c|}{ Heart rate (HR) (beats/min) } \\
\hline Baseline & $81.5 \pm 8.3$ & $81.3 \pm 8.4$ & $82.3 \pm 8.1$ & $82.2 \pm 8.2$ \\
\hline 10 min_Anesthesia & $67.5 \pm 6.2^{\mathrm{a}}$ & $65.7 \pm 7.1^{\mathrm{a}}$ & $66.5 \pm 7.4^{\mathrm{a}}$ & $68.8 \pm 7.8^{\mathrm{a}}$ \\
\hline 30 min_Anesthesia & $69.7 \pm 8.2^{a}$ & $66.9 \pm 8.7^{\mathrm{a}}$ & $66.6 \pm 8.9^{\mathrm{a}}$ & $67.9 \pm 8.7^{\mathrm{a}}$ \\
\hline 60 min_Anesthesia & $71.3 \pm 8.4^{\mathrm{a}}$ & $68.5 \pm 8.9^{a}$ & $68.8 \pm 8.7^{\mathrm{a}}$ & $71.5 \pm 8.9^{\mathrm{a}}$ \\
\hline End of operation & $71.5 \pm 8.6^{a}$ & $71.5 \pm 8.5^{\mathrm{a}}$ & $71.3 \pm 8.9^{\mathrm{a}}$ & $71.6 \pm 9.1^{\mathrm{a}}$ \\
\hline \multicolumn{5}{|c|}{ Rate pressure product (RPP) } \\
\hline Baseline & $10,451.3 \pm 269.5$ & $10,487.6 \pm 281.7$ & $10,490.2 \pm 290.8$ & $10,498.6 \pm 297.3$ \\
\hline 10 min_Anesthesia & $7572.7 \pm 187.6^{\mathrm{a}}$ & $9695.4 \pm 295.4^{c}$ & $9605.6 \pm 301^{c}$ & $9612.9 \pm 308.5^{c}$ \\
\hline 30 min_Anesthesia & $7691.3 \pm 279.4^{\mathrm{a}}$ & $9595.5 \pm 297.3^{c}$ & $9609.8 \pm 306.5^{c}$ & $9925.7 \pm 315.7^{c}$ \\
\hline 60 min_Anesthesia & $7718.6 \pm 286.2^{\mathrm{a}}$ & $9601.7 \pm 305.7^{c}$ & $10,313.5 \pm 287.3^{c}$ & $13,076.5 \pm 356.2^{\text {acef }}$ \\
\hline End of operation & $7995.4 \pm 297.3^{\mathrm{a}}$ & $9611.8 \pm 308.9^{c}$ & $10,482.7 \pm 291.5^{c}$ & $13,218.6 \pm 395.8^{\text {acef }}$ \\
\hline \multicolumn{5}{|c|}{ Central venous pressure (CVP) $\left(\mathrm{cmH}_{2} \mathrm{O}\right)$} \\
\hline Baseline & $8.6 \pm 1.2$ & $8.7 \pm 1.2$ & $8.5 \pm 1.3$ & $8.6 \pm 1.3$ \\
\hline 10 min_Anesthesia & $3.9 \pm 1.1^{\mathrm{a}}$ & $5.7 \pm 1.0^{\mathrm{ac}}$ & $5.8 \pm 1.1^{\mathrm{ac}}$ & $6.2 \pm 1.1^{\mathrm{ac}}$ \\
\hline 30 min_Anesthesia & $4.0 \pm 1.1^{\mathrm{a}}$ & $6.0 \pm 1.1^{\mathrm{ac}}$ & $6.1 \pm 1.0^{\mathrm{ac}}$ & $6.4 \pm 1.1^{\mathrm{ac}}$ \\
\hline 60 min_Anesthesia & $4.5 \pm 1.0^{\mathrm{a}}$ & $6.1 \pm 1.0^{\mathrm{ac}}$ & $6.3 \pm 1.1^{\mathrm{ac}}$ & $8.5 \pm 1.2^{\text {cef }}$ \\
\hline End of operation & $4.7 \pm 1.1^{\mathrm{a}}$ & $6.3 \pm 1.1^{\mathrm{ac}}$ & $6.5 \pm 1.0^{\mathrm{ac}}$ & $8.7 \pm 1.3^{\mathrm{cef}}$ \\
\hline \multicolumn{5}{|c|}{ Saturation of peripheral oxygen $\left(\mathrm{SpO}_{2}\right)(\%)$} \\
\hline Baseline & $98.6 \pm 1.1$ & $98.7 \pm 1.1$ & $98.8 \pm 1.0$ & $98.7 \pm 1.2$ \\
\hline 10 min_Anesthesia & $98.1 \pm 1.1$ & $98.4 \pm 1.1$ & $98.6 \pm 1.2$ & $98.6 \pm 1.1$ \\
\hline 30 min_Anesthesia & $98.5 \pm 1.2$ & $98.6 \pm 1.0$ & $98.7 \pm 1.2$ & $98.7 \pm 1.0$ \\
\hline 60 min_Anesthesia & $98.4 \pm 1.0$ & $98.8 \pm 1.1$ & $98.7 \pm 1.1$ & $98.8 \pm 1.1$ \\
\hline End of operation & $98.3 \pm 1.3$ & $98.8 \pm 1.1$ & $98.8 \pm 1.0$ & $98.8 \pm 1.1$ \\
\hline \multicolumn{5}{|c|}{ Cardiac output (CO) (L/min) } \\
\hline Baseline & $6.99 \pm 1.12$ & $6.98 \pm 1.16$ & $7.07 \pm 1.12$ & $7.08 \pm 1.12$ \\
\hline 10 min_Anesthesia & $4.44 \pm 0.84^{\mathrm{a}}$ & $6.06 \pm 1.13^{c}$ & $6.21 \pm 1.17^{c}$ & $6.44 \pm 1.24^{c}$ \\
\hline 30 min_Anesthesia & $4.68 \pm 1.00^{\mathrm{a}}$ & $6.31 \pm 1.30^{c}$ & $6.30 \pm 1.31^{c}$ & $6.47 \pm 1.33^{c}$ \\
\hline 60 min_Anesthesia & $4.93 \pm 1.05^{\mathrm{a}}$ & $6.48 \pm 1.35^{c}$ & $6.52 . \pm 1.3^{c}$ & $9.04 \pm 1.81^{\text {acef }}$ \\
\hline End of operation & $4.96 \pm 1.08^{\mathrm{a}}$ & $6.78 \pm 1.34^{c}$ & $6.78 \pm 1.39^{c}$ & $9.41 \pm 1.91^{\text {acef }}$ \\
\hline \multicolumn{5}{|c|}{ Systolic volume (SV) (mL/beat) } \\
\hline Baseline & $85.3 \pm 5.0$ & $85.3 \pm 5.4$ & $85.4 \pm 5.2$ & $85.6 \pm 5.1$ \\
\hline 10 min_Anesthesia & 65.3. $\pm 6.4^{\mathrm{a}}$ & $91.2 \pm 7.1^{c}$ & $92.5 \pm 7.3^{c}$ & $92.7 \pm 7.4^{c}$ \\
\hline 30 min_Anesthesia & $66.4 \pm 6.5^{\mathrm{a}}$ & $93.4 \pm 7.3^{c}$ & $93.6 \pm 7.2^{c}$ & $94.2 \pm 7.5^{c}$ \\
\hline 60 min_Anesthesia & $68.3 \pm 6.7^{a}$ & $93.6 \pm 7.5^{c}$ & $93.8 \pm 7.3^{c}$ & $125.2 \pm 9.7^{\text {acef }}$ \\
\hline End of operation & $68.6 \pm 6.9^{a}$ & $93.9 \pm 7.6^{c}$ & $94.1 \pm 7.8^{c}$ & $130.2 \pm 10.1^{\text {acef }}$ \\
\hline
\end{tabular}

Data are presented as mean \pm standard deviation (SD)

${ }^{\mathrm{a}} P<0.05$, compared with that at $10 \mathrm{~min}$ before anesthesia induction

${ }^{\mathrm{c}} P<0.05$, compared with the control group (C)

e $P<0.05$, compared with the M1 group

${ }^{f} P<0.05$, compared with the M2 group 
that at baseline), low blood pressure $(<70 \%$ of that at baseline), tachycardia (HR > 100 beats/min), and bradycardia (HR $<60$ beats/min) during surgery were counted, and the accumulated duration of low blood pressure was calculated. In cases of either high or low blood pressure, patients were immediately treated with either continuous intravenous nitroglycerin infusion at $1-3 \mu \mathrm{g} \cdot \mathrm{kg}^{-1} \cdot \mathrm{min}^{-1}$ or an increased transfusion rate and intravenous injection of ephedrine (5-6 mg per patient), respectively. Tachycardia and bradycardia were treated with immediate intravenous injection of esmolol hydrochloride (10-20 mg) or atropine $(0.5-1.0 \mathrm{mg})$, respectively. When the $\mathrm{SpO}_{2}$ was lower than $90 \%$, the oxygen pressure in the inspired air was increased.

\section{Use of analgesics}

All patients received sufentanil and ropivacaine for pain control after surgery. In each patient, sufentanil (30 mcg) mixed with $0.2 \%$ ropivacaine and diluted in a total volume of $100 \mathrm{~mL}$ was administered via a pump (Royal Fornia Medical, Zhuhai, China) for patient-controlled epidural analgesia (PCEA) for $48 \mathrm{~h}$. The pump was programmed to deliver drugs in a $0.5-\mathrm{mL}$ bolus with a lockout time of $15 \mathrm{~min}$ and a background infusion rate of $2 \mathrm{~mL} / \mathrm{h}$ [21].

\section{Assessment of postoperative analgesia quality}

Analgesia quality and comfort level were assessed at 1 , $6,12,18,24,36$, and $48 \mathrm{~h}$ postoperation. The analgesia quality was assessed using the visual analogue scale (VAS) with 0 for no pain, 10 points for drastic pain, $>5$ points for unsatisfactory analgesia, 3-4 points for acceptable analgesia, and $<3$ points for satisfactory analgesia [22]. The comfort level was evaluated using the Bruggrmann Comfort Scale (BCS) with 0 for persistent pain, 1 for severe pain while moving the operated limb, 2 for mild pain while moving the operated limb, 3 for no pain while moving the operated limb, and 4 for no pain while slightly touching the incision [23].

\section{Assessment of cognitive function}

The MMSE has been extensively used in clinical and research settings for assessing cognitive impairment. It is a simple, practical, and highly reliable 30-point questionnaire that excludes the influences of emotional and mental disorders on cognitive function [24]. The MMSE test was conducted in each patient 1 day before and 1, 3, and 7 days after surgery by the same psychologist. The functions that were examined included orientation (temporal orientation, 5 points; spatial orientation, 5 points), recall (immediate recall, 3 points; delayed recall, 3 points), language (articulation, 2 points; recitation, 1 point; writing, 1 point), execution (ability to follow simple commands, 1 point), and calculation (5 points). POCD was defined as decreases in MMSE test scores by more than 2 points from scores before surgery [24].

\section{Measurement of blood TNF-a level}

Blood samples were collected from the nontransfused forearm ( $3 \mathrm{~mL}$ per patient) at $10 \mathrm{~min}$ before anesthesia, at the end of surgery, and at 1 and 2 days after surgery on an empty stomach. After collection, the samples were immediately dissolved in $5 \mathrm{~mL}$ ethylene diamine tetraacetic acid. The solution was then centrifuged in a Beckman Allegra TM21 R centrifuge at $3000 \mathrm{r} / \mathrm{min}$ for $5 \mathrm{~min}$. Then the supernatant serum was separated and stored at $4^{\circ} \mathrm{C}$. An ELISA kit (Shanghai Xinyu Biology, Ltd) was used to measure the TNF- $\alpha$ concentration.

\section{Sample size estimation and statistical analysis}

The sample size was estimated for detecting differences in the primary outcome variable (i.e., incidence of POCD) using Power Analysis and Sample Size 11.0 (PASS 11.0) with $90 \%$ power $(1-\beta)$ and $\alpha=0.05$ (two-sided). According to and results from a pilot study, the incidence rates of POCD were found to be $4 \%, 10 \%, 15 \%$, and $25 \%$ in a control group and low-dose, moderate-dose, and high-dose methoxamine groups, respectively. The effect size, i.e., the minimum detectable difference among the four groups, was calculated to be 0.225 based on a contingency table [20], and the minimum sample size was found to be 280 for all four groups ( $n=70$ in each group) according to Chi-square test for multiple proportions. Considering some participants might drop out, the final sample size was set at $n=75$ for each group.

Statistical analyses of inter-group differences were performed using SPSS 15.0 (SPSS Inc., Chicago, IL, USA).

Table 3 Incidences of cardiovascular complications and duration of low blood pressure (LBP) during surgery in different groups

\begin{tabular}{|c|c|c|c|c|c|c|}
\hline \multirow[t]{2}{*}{ Group } & $\mathrm{HBP}$ & LBP & Tachycardia & Bradycardia & Pulmonary edema & \multirow{2}{*}{$\begin{array}{l}\text { Accumulated duration } \\
\text { of } L B P \text {, min }\end{array}$} \\
\hline & \multicolumn{5}{|l|}{$n(\%)$} & \\
\hline$C$ & $0(0)$ & $16(21.3)$ & $0(0)$ & $19(25.3)$ & $2(2.7)$ & $2.3 \pm 0.5$ \\
\hline M1 & $0(0)$ & $0(0)^{a}$ & $0(0)$ & $16(21.3)$ & $0(0)^{b}$ & $0^{b}$ \\
\hline M2 & $0(0)$ & $0(0)^{a}$ & $0(0)$ & $18(24.0)$ & $0(0)^{b}$ & $0^{b}$ \\
\hline M3 & $32(42.6)^{\mathrm{a}}$ & $0(0)^{a}$ & $0(0)$ & $17(22.7)$ & $0(0)^{b}$ & $0^{\mathrm{b}}$ \\
\hline
\end{tabular}

$H B P$ high blood pressure

a $P<0.01$

${ }^{\mathrm{b}} P<0.05$, compared with the control group (C) 


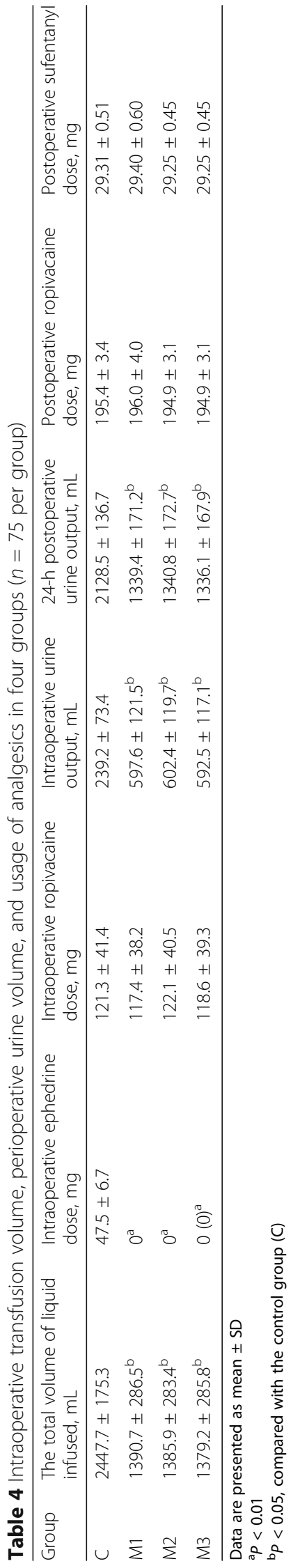


All numerical values are presented as mean \pm standard deviation (SD). Repeated measures ANOVA were used to compare intra-group differences. One-way ANOVA and post-hoc tests were performed to compare inter-group differences. Categorical data were compared using the chisquare test. Statistical significance was accepted at $P<0.05$.

\section{Results}

The incidence of POCD was the primary outcome of this study. The general clinical information, operation time, and red blood cell count for the four study groups are presented in Table 1. There were no statistical differences in any of the parameters among the groups $(P>0.05)$.

Table 2 summarizes the hemodynamic parameters at different time points in the four groups. As compared with those measured $10 \mathrm{~min}$ before anesthesia (T1), the control group showed decreased MAP, CVP, HR, RPP, $\mathrm{CO}$, and SV; all methoxamine treatment groups (M1, M2, and M3) showed a decreased HR; the M1 and M2 groups showed a decreased CVP at all time points (T2, T3, and T4) after anesthesia and at the end of surgery (T5); and M3 group showed increased MAP, RPP, CO, and SV at T4 and T5 $(P<0.05)$. As compared with the control group, all methoxamine treatment groups had higher MAP, CVP, RPP, CO, and SV values at T2, T3, T4, and T5 (all $P<0.05$ ). At T4 and T5, the M3 group had even higher MAP, CVP, RPP, CO, and SV values than the M1 and M2 groups (all $P<0.05$ ).

Table 3 summarizes the incidences of cardiovascular complications during surgery in the different groups. Compared with the control group, all methoxamine groups had a lower incidence and shorter duration of low blood pressure, whereas the M3 group had a higher incidence of high blood pressure $(P<0.01$ or $<0.05)$.

Table 4 summarizes the intraoperative liquid transfusion volume, perioperative urine volume, and drug usage in the four groups. Compared with the control group, all methoxamine treatment groups had significantly lower intraoperative liquid transfusion volume, less ephedrine usage, higher intraoperative urine volume, and lower postoperative $24 \mathrm{~h}$ urine volume $(P<0.01$ or $<0.05)$. No statistical differences in ropivacaine and sufentanil usage were observed among the four groups $(P>0.05)$

Table 5 shows the results of assessments of analgesia quality and comfort level using the VAS and BCS, respectively. Satisfactory analgesia was achieved at all time points in all groups, with no statistical differences observed $(P>0.05)$.

As shown in Table 6, MMSE test scores were lower at 1 and 3 days after operation in the M3 group than in the control group and were lower in both of these groups than those at 1 day before surgery $(P<0.05)$, with the M3 group showing a lower mean score than the control group $(P<0.05)$. Meanwhile, the incidence of POCD was remarkably higher in the M3 group (18.7\%) than in the control (5.3\%), M1 (6.7\%), and M2 (6.7\%) groups $(P<0.05)$, compared with that at $10 \mathrm{~min}$ before anesthesia (baseline; $P<0.05$ ). As shown in Table 7 , blood TNF- $\alpha$ levels at the end of surgery and 1 and 2 days after surgery were higher in the M3 group than in the control, M1, and M2 groups and were higher than the respective baseline levels in all groups $(P<0.05)$.

\section{Discussion}

In this study, at all studying time points during anesthesia, the M1 and M2 groups only showed decreases in the CVP and HR, whereas the control group exhibited significant decreases in various hemodynamic parameters. Meanwhile, compared with the control group, all methoxamine treatment groups required less transfusion volume, showed increased urine excretion during surgery and decreased urine excretion after surgery, suggesting less urine retention. On the other hand, as the surgical time increased, intravenous infusion of methoxamine at $4 \mu \mathrm{g} \cdot \mathrm{kg}^{-1} \cdot \mathrm{min}^{-1}$ caused increases in blood pressure (in up to $42.6 \%$ of the 75 patients), cardiac output, and systolic stroke volume, leading to increased myocardium oxygen consumption. These results demonstrated that intravenous infusion of methoxamine at an adequate dosage $\left(2-3 \mu \mathrm{g} \cdot \mathrm{kg}^{-1} \cdot \mathrm{min}^{-1}\right)$ could provide better control of the hemodynamics during epidural anesthesia and surgery. The decreases in CVP in all

Table 5 Assessment of analgesia quality and comfort level at multiple postoperative time points in four groups ( $n=75$ per group)

\begin{tabular}{|c|c|c|c|c|c|c|c|c|}
\hline & Group & $1 \mathrm{~h}$ & $6 \mathrm{~h}$ & $12 \mathrm{~h}$ & $18 \mathrm{~h}$ & $24 \mathrm{~h}$ & $36 \mathrm{~h}$ & $48 \mathrm{~h}$ \\
\hline \multirow[t]{4}{*}{$\overline{\text { VAS }}$} & $C$ & $1.75 \pm 0.13$ & $1.77 \pm 0.13$ & $1.77 \pm 0.12$ & $1.76 \pm 0.13$ & $1.75 \pm 0.12$ & $1.76 \pm 0.12$ & $1.77 \pm 0.11$ \\
\hline & M1 & $1.76 \pm 0.12$ & $1.78 \pm 0.14$ & $1.76 \pm 0.13$ & $1.77 \pm 0.14$ & $1.76 \pm 0.13$ & $1.77 \pm 0.11$ & $1.78 \pm 0.12$ \\
\hline & M2 & $1.75 \pm 0.12$ & $1.77 \pm 0.13$ & $1.78 \pm 0.11$ & $1.77 \pm 0.12$ & $1.76 \pm 0.14$ & $1.77 \pm 0.13$ & $1.78 \pm 0.14$ \\
\hline & M3 & $1.77 \pm 0.11$ & $1.76 \pm 0.12$ & $1.76 \pm 0.13$ & $1.76 \pm 0.14$ & $1.75 \pm 0.13$ & $1.76 \pm 0.11$ & $1.78 \pm 0.11$ \\
\hline \multirow[t]{4}{*}{$\mathrm{BCS}$} & C & $3.88 \pm 0.10$ & $3.86 \pm 0.12$ & $3.87 \pm 0.09$ & $3.87 \pm 0.07$ & $3.87 \pm 0.08$ & $3.89 \pm 0.10$ & $3.88 \pm 0.07$ \\
\hline & $\mathrm{M} 1$ & $3.86 \pm 0.12$ & $3.85 \pm 0.13$ & $3.87 \pm 0.12$ & $3.86 \pm 0.10$ & $3.86 \pm 0.11$ & $3.88 \pm 0.11$ & $3.89 \pm 0.10$ \\
\hline & M2 & $3.87 \pm 0.13$ & $3.87 \pm 0.10$ & $3.86 \pm 0.10$ & $3.87 \pm 0.10$ & $3.87 \pm 0.08$ & $3.87 \pm 0.10$ & $3.88 \pm 0.09$ \\
\hline & M3 & $3.87 \pm 0.11$ & $3.86 \pm 0.13$ & $3.86 \pm 0.11$ & $3.86 \pm 0.12$ & $3.87 \pm 0.12$ & $3.88 \pm 0.11$ & $3.88 \pm 0.10$ \\
\hline
\end{tabular}

Data are presented as mean \pm SD. No statistical difference was observed 
Table 6 MMSE test scores in four groups at different time points ( $n=75$ per group)

\begin{tabular}{llllll}
\hline Group & $1 \mathrm{~d}$ before operation & $1 \mathrm{~d}$ post operation & $3 \mathrm{~d}$ post operation & $7 \mathrm{~d}$ post operation & POCD, $n(\%)$ \\
\hline C & $27.8 \pm 2.1$ & $24.3 \pm 2.5^{\mathrm{a}}$ & $25.2 \pm 2.4^{\mathrm{a}}$ & $27.6 \pm 2.2$ & $4(5.3)$ \\
M1 & $27.7 \pm 2.1$ & $24.5 \pm 2.4^{\mathrm{ac}}$ & $24.9 \pm 2.5^{\mathrm{a}}$ & $27.5 \pm 2.2$ & $5(6.7)$ \\
M2 & $27.9 \pm 2.1$ & $25.1 \pm 2.5^{\mathrm{ac}}$ & $25.3 \pm 2.3^{\mathrm{a}}$ & $27.6 \pm 2.3$ & $5(6.7)$ \\
M3 & $27.8 \pm 2.0$ & $22.2 \pm 2.4^{\mathrm{ac}}$ & $22.8 \pm 2.5^{\mathrm{ac}}$ & $27.4 \pm 2.0$ & $14(18.7)^{\mathrm{c}}$ \\
\hline
\end{tabular}

Data are presented as mean \pm SD

${ }^{a} P<0.05$, compared with 1 day before operation

${ }^{c} P<0.05$, compared with the control group

groups were mainly due to dilation of peripheral resistance vessels and venous vessels, caused by sympathetic block associated with epidural anesthesia. The high incidence of bradycardia in all groups may be the result of increased activity of the vagus nerve due to epidural anesthesia, which on the other hand further confirms that methoxamine has the potential to regulate vagus nerve activity via the carotid sinus baroreflex, reducing myocardium oxygen consumption while maintaining a high blood pressure $[17,18]$. The concomitant low blood pressure (in $21.3 \%$ of the 75 patients) and pulmonary edema (2.7\%) may be caused by the dilation of peripheral vessels and excessive liquid transfusion in efforts to maintain a normal blood volume.

Surgical insults can activate the peripheral immune system and cause inflammation, leading to the excretion of various inflammatory cytokines such as interleukin$1 \beta$, interleukin- 6 , and TNF- $\alpha$, which can be transported through the blood-brain barrier and activate astrocytes and microglia to induce the cytotoxic reaction and damage cognitive function $[9,10,25,26]$. In elderly rats with POCD, microglia and neuronal dendrites were observed to be significantly activated in the hippocampus, accompanied by dramatic increases of TNF- $\alpha$ and IL- $1 \beta$ levels, suggesting that POCD probably originates from neuroinflammation in the hippocampus [27]. In the present study, all groups showed higher TNF- $\alpha$ levels after surgery compared with baseline levels, and the M3 group had a higher TNF- $\alpha$ level than the control, M1, and M2 groups. Meanwhile, the incidence of POCD was observed to be much higher in the M3 group (18.7\%) than in the control (5.3\%), M1 (6.7\%), and M2 (6.7\%) groups.
These results support that POCD can be associated with excessive excretion of TNF- $\alpha$, consistent with the findings reported by the above-described literature. In the control group, the incidence of POCD was relatively low, though $21.3 \%$ of the patients presented with low blood pressure. This was possibly because the accumulative duration of low blood pressure was actually short in these patients $(2.3 \pm 0.5 \mathrm{~min})$ due to timely intervention.

Benzodiazepines, anticholinergic agents, pain, and hypoxemia also can increase the risk of developing POCD [28-31]. In this study, the usage of benzodiazepines and anticholinergic agents were avoided in all groups both before and after surgery. The intraoperative CVP and $\mathrm{SpO}_{2}$ were in the normal ranges in all patients. Satisfactory analgesia was achieved in all patients. These facts also to some extent helped to reduce the potential risk of developing POCD.

The major limitation of this study is that as compared with patients who received lower doses, the patients who received methoxamine at the dose of $4 \mu \mathrm{g} \cdot \mathrm{kg}^{-1} \cdot \mathrm{min}^{-1}$ did not show further improved intraoperative hemodynamic stability. Furthermore, these patients underwent elevated myocardial oxygen consumption and showed higher incidence of POCD. Future studies should include compensation measures for unexpected loss in the patients.

\section{Conclusion}

In conclusion, intravenous infusion of methoxamine at 2$3 \mu \mathrm{g} \cdot \mathrm{kg}^{-1} \cdot \mathrm{min}^{-1}$ was demonstrated effective in maintaining stable hemodynamics in elderly patients during epidural anesthesia for hip-joint replacement surgery, without increasing the incidence of POCD.

Table 7 Serum TNF-a levels (pg/mL) in four groups at different time points ( $n=75$ per group)

\begin{tabular}{lllll}
\hline Group & Baseline & End of operation & $24 \mathrm{~h}$ after operation & $48 \mathrm{~h}$ after operation \\
\hline C & $12.64 \pm 3.21$ & $31.95 \pm 6.19^{\mathrm{a}}$ & $32.12 \pm 6.59^{\mathrm{a}}$ & $35.09 \pm 6.21^{\mathrm{a}}$ \\
M1 & $13.09 \pm 3.14$ & $32.09 \pm 6.20^{\mathrm{a}}$ & $33.17 \pm 7.35^{\mathrm{a}}$ & $36.11 \pm 6.23^{\mathrm{a}}$ \\
M2 & $12.08 \pm 3.22$ & $34.15 \pm 6.25^{\mathrm{a}}$ & $36.19 \pm 6.54^{\mathrm{a}}$ & $39.15 \pm 7.17^{\mathrm{a}}$ \\
M3 & $12.11 \pm 3.19$ & $58.08 \pm 6.21^{\mathrm{abcd}}$ & $61.7 \pm 13.31^{\mathrm{abcd}}$ & $64.14 \pm 14.19^{\mathrm{abcd}}$ \\
\hline
\end{tabular}

Data are presented as mean \pm SD

${ }^{a} P<0.05$, compared with that at baseline

${ }^{\mathrm{b}} P<0.05$, compared with the $\mathrm{M} 1$ group

${ }^{c} P<0.05$, compared with the control group

${ }^{\mathrm{d}} p<0.05$, compared with the $\mathrm{M} 2$ group 


\section{Abbreviations}

ASA: American Society of Anesthesiologists; MMSE: Mini-mental state examination; POCD: Postoperative cognitive dysfunction; TNF-a: Tumor necrosis factor- $a$

\section{Acknowledgements}

None.

\section{Funding}

None.

\section{Availability of data and materials}

The datasets created during and/or analyzed during the current study available from the corresponding author on reasonable request.

\section{Authors' contributions}

SDF conceived the experiment; SSH and $\mathrm{HJ}$ collected the data; $\mathrm{WLJ}, \mathrm{YL}$ and LRC analyzed the data; SSH and SDF wrote the paper. All authors read and approved the final manuscript.

\section{Competing interests}

The authors declare that they have no competing interests.

\section{Consent for publication}

Not applicable.

\section{Ethics approval and consent to participate}

This study was preapproved by our institutional Research Ethics Committee in 2012 (Identification No.:KY2012-42). Written informed consent was obtained from each patient or his/her authorized representative(s) if he/she was in a critical physical condition or mentally challenged.

\section{Publisher's Note}

Springer Nature remains neutral with regard to jurisdictional claims in published maps and institutional affiliations.

\section{Author details}

${ }^{1}$ Affiliated High School of Liaoning Normal University, Dalian, People's Republic of China. 'Department of Anesthesiology, The First Affiliated Hospital of Dalian Medical University, No.222 ZhongshanRoad, Xigang District, Dalian 116011, Liaoning, People's Republic of China. ${ }^{3}$ Department of Neuroelectrophysiology, The First Affiliated Hospital of Dalian Medical University, Dalian, People's Republic of China.

\section{Received: 28 February 2017 Accepted: 28 May 2017}

Published online: 09 June 2017

\section{References}

1. Bryson GL, Wyand A. Evidence-based clinical update: general anesthesia and the risk of delirium and postoperative cognitive dysfunction. Can J Anaesth. 2006;53(7):669-77. doi:10.1007/BF03021625.

2. Moller JT, Cluitmans P, Rasmussen LS, et al. Long-term postoperative cognitive dysfunction in the elderly ISPOCD1 study. ISPOCD investigators. International study of post-operative cognitive dysfunction. Lancet. 1998; 351(9106):857-61.

3. Morandi A, Pandharipande PP, Jackson JC, et al. Understanding terminology of delirium and long-term cognitive impairment in critically ill patients. Best Pract Res Clin Anaesthesiol. 2012;26(3):267-76. doi:10.1016/j.bpa.2012.08.001.

4. Selnes OA, McKhann GM. Neurocognitive complications after coronary artery bypass surgery. Ann Neurol. 2005;57(5):615-21. doi:10.1002/ana.20481.

5. Rundshagen I. Postoperative cognitive dysfunction. Dtsch Arztebl Int. 2014; 111(8):119-25. doi:10.3238/arztebl.2014.0119.

6. $\mathrm{Xu} \mathrm{Z}$, Dong $\mathrm{Y}$, Wang $\mathrm{H}$, et al. Age-dependent postoperative cognitive impairment and Alzheimer-related neuropathology in mice. Sci Rep. 2014;4: 3766. doi:10.1038/srep03766

7. Zhou F, Li ZD, Yu JB. The research progress in Pathophysiology of postoperative cognitive dysfunction. Med Recapitulate (China). 2011;17(7): 1026-9.

8. Lin SY, Gao J. A new research progress on postoperative cognitive dysfunction in elderly patients. Guangdong Med J (China). 2010:31(2):243-4.

9. Sheeran P, Hall GM. Cytokines in anaesthesia. Br J Anaesth. 1997;78(2):201-19.
10. Wilson CJ, Finch CE, Cohen HJ. Cytokines and cognition-the case for a head-to-toe inflammatory paradigm. J Am Geriatr Soc. 2002:50(12):2041-56.

11. Oberbeck R. Catecholamines: physiological immunomodulators during health and illness. Curr Med Chem. 2006;13(17):1979-89.

12. Markus T, Hansson SR, Cronberg T, et al. beta-Adrenoceptor activation depresses brain inflammation and is neuroprotective in lipopolysaccharideinduced sensitization to oxygen-glucose deprivation in organotypic hippocampal slices. J Neuroinflammation. 2010;7:94. doi:10.1186/1742-2094-7-94.

13. Shi HJ, Xue XH, Wang YL, et al. Effects of different anesthesia methods on cognitive dysfunction after hip replacement operation in elder patients. Int J Clin Exp Med. 2015:8(3):3883-8.

14. Barre J, Lepouse C, Segal P. Embolism and intramedullary femoral surgery. Rev Chir Orthop Reparatrice Appar Mot. 1997;83(1):9-21.

15. Rooke GA, Freund PR, Jacobson AF. Hemodynamic response and change in organ blood volume during spinal anesthesia in elderly men with cardiac disease. Anesth Analg. 1997;85(1):99-105.

16. Kasaba T, Yamaga M, Iwasaki T, et al. Ephedrine, dopamine, or dobutamine to treat hypotension with propofol during epidural anesthesia. Can J Anaesth. 2000;47(3):237-41.

17. Thiele RH, Nemergut EC, Lynch C 3rd. The clinical implications of isolated alpha(1) adrenergic stimulation. Anesth Analg. 2011;113(2):297-304. doi:10. 1213/ANE.0b013e3182120ca5.

18. Mercier FJ, Riley ET, Frederickson WL, et al. Phenylephrine added to prophylactic ephedrine infusion during spinal anesthesia for elective cesarean section. Anesthesiology. 2001;95(3):668-74.

19. Sun $D, W u Y$, Yang $L$, et al. Effects of continuous intravenous infusion of methoxamine on the intraoperative hemodynamics of elderly patients undergoing total hip arthroplasty. Med Sci Monit. 2014;20:1969-76. doi:10.12659/MSM.890760

20. Sun $D, W u Y$, Lin $Y$, et al. Effect of intravenous infusion of dobutamine hydrochloride on the development of early postoperative cognitive dysfunction in elderly patients via inhibiting the release of tumor necrosis factor-a. Eur J Pharmacol. 2014:741:150-5.

21. Xu GH, Gao M, Sheng QY, et al. Opioid receptor A118G polymorphism does not affect the consumption of sufentanil and ropivacaine by patientcontrolled epidural analgesia after cesarean section. Ther Drug Monit. 2015; 37(1):53-7. doi:10.1097/ftd.0000000000000112

22. Rauh $\mathrm{KH}$, Andersen RS, Rosenberg J. Visual analogue scale for measuring post-operative pain. Ugeskr Laeger. 2013;175(24):1712-6.

23. Vasseur E, Gibbons J, Rushen J, et al. Development and implementation of a training program to ensure high repeatability of body condition scoring of dairy cows. J Dairy Sci. 2013:96(7):4725-37. doi:10.3168/jds.2012-6359.

24. Anthony JC, LeResche L, Niaz U, et al. Limits of the 'Mini-mental State' as a screening test for dementia and delirium among hospital patients. Psychol Med. 1982;12(2):397-408.

25. Hovens IB, Schoemaker RG, van der Zee EA, et al. Postoperative cognitive dysfunction: involvement of neuroinflammation and neuronal functioning Brain Behav Immun. 2014:38:202-10. doi:10.1016/j.bbi.2014.02.002.

26. Krenk L, Rasmussen LS, Kehlet H. New insights into the pathophysiology of postoperative cognitive dysfunction. Acta Anaesthesiol Scand. 2010;54(8): 951-6. doi:10.1111/j.1399-6576.2010.02268.x.

27. Li Y, Wang S, Ran K, et al. Differential hippocampal protein expression between normal aged rats and aged rats with postoperative cognitive dysfunction: a proteomic analysis. Mol Med Rep. 2015;12(2):2953-60. doi:10.3892/mmr.2015.3697.

28. Fujita $Y$, Nakamura $K$, Horiguchi $Y$, et al. Effect of different perioperative analgesic methods on postoperative cognitive dysfunction in elderly patients undergoing upper abdominal surgery. Masui. 2011;60(10):1153-8.

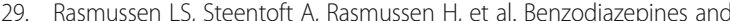
postoperative cognitive dysfunction in the elderly. ISPOCD group. International study of postoperative cognitive dysfunction. $\mathrm{Br} J$ Anaesth. 1999:83(4):585-9.

30. Rossi A, Burkhart C, Dell-Kuster S, et al. Serum anticholinergic activity and postoperative cognitive dysfunction in elderly patients. Anesth Analg. 2014; 119(4):947-55. doi:10.1213/ANE.0000000000000390.

31. Xu JH, Zhang TZ, Peng XF, et al. Effects of sevoflurane before cardiopulmonary bypass on cerebral oxygen balance and early postoperative cognitive dysfunction. Neurol Sci. 2013;34(12):2123-9. doi:10.1007/s10072-013-1347-3. 